\title{
FINITE ELEMENT ANALYSIS OF STRAIN EFFECTS ON ELECTRONIC AND TRANSPORT PROPERTIES IN QUANTUM DOTS AND WIRES
}

\author{
H. T. Johnson, L. B. Freund, C. D. Akyüz, and A. Zaslavsky \\ Division of Engineering, Brown University, Providence, RI 02912
}

\begin{abstract}
Lattice mismatch in epitaxial layered heterostructures with small characteristic lengths induces large, spatially nonuniform strains. The components of the strain tensor have been shown experimentally to affect the electronic properties of semiconductor structures. Here a technique is presented for calculating the influence of strain on electronic properties. First the linear elastic strain in a quantum dot or wire is determined by a finite element calculation. A strain-induced potential field that shifts and couples the valence subbands in the structure is then determined from deformation potential theory. The time-independent Schrödinger equation, including the nonuniform strain induced potential and a potential due to the heterostructure layers, is then solved, also by means of the finite element method. The solution consists of the wavefunctions and energies of states confined to the active region of the structure; these are the features which govern the electronic and transport properties of devices. As examples, two $\mathrm{Si}_{x} \mathrm{Ge}_{1-x}$ submicron resonant tunneling devices, a quantum wire with two dimensional confinement and a quantum dot with three dimensional confinement, are analyzed. Experimentally measured resonant tunneling current peaks corresponding to the valence subbands in the material are modeled by generating densities of confined states in the structures. Size and composition dependent strain effects are examined for both devices. In both the quantum dot and the quantum wire, the strain effects on the wavefunctions and energies of confined states are evident in the calculated densities of confined states in the structures, which are found to be consistent with experimentally measured tunneling current/voltage curves for resonant tunneling diodes.
\end{abstract}




\section{INTRODUCTION}

Epitaxially grown semiconductor heterostructures often consist of materials with lattice parameters that are mismatched by as much as several percent. For thin films of large lateral extent, these strains are spatially uniform and the effects are well understood. However, structures of relatively small lateral extent, having distinctive geometric features and bounded by free surfaces, are characterized by strains that are highly nonuniform. The effects of nonuniform strain on the electronic properties of semiconductor heterostructures have been observed experimentally, but the coupled physical phenomenon has not been extensively modeled. ${ }^{1-4}$ The analysis of strain effects in a quantum mechanical model of semiconductor devices has only recently been attempted by Pryor et al. ${ }^{5-7}$ and Williamson et al. ${ }^{8}$, who calculate strain induced potentials and wavefunctions in quantum dots, and Zunger ${ }^{9}$, who reviews the topic of electronic structure in pyramidal semiconductor quantum dots based on atomistic methods.

Strained semiconductor devices that are based on quantum effects, particularly charge confinement in one or more spatial dimensions, underlie a potentially significant technology. Much can be learned about quantum effects by studying the class of devices based on resonant tunneling of carriers from an emitter region, into a quantum well, and then into a collector region. The material combinations in these devices and their geometrical features, including the layered structure and the free surfaces, lead to complicated mechanical strain fields. Because the sequential tunneling of carriers is a simple phenomenon governed by the spectrum of available states in the quantum well, and because the devices are extremely small and the operating temperatures are low, it is likely that the effects of strain on the electronic and transport properties can be represented quantitatively through modeling.

Calculations of elastic strain fields in semiconductor structures are well suited for the finite element method, which is a common tool in continuum mechanics. ${ }^{2,10}$ In the technique presented here, the finite element calculation of the strain in a device is made using a general purpose structural mechanics finite element package. ${ }^{11}$ However, the use of the finite element method (FEM) in quantum mechanics, which is reviewed by Linderberg ${ }^{12}$, is much less common. Models of semiconductor devices by means of FEM have been proposed by a number of authors for one and two dimensional problems. Several FEM models are available for one dimensional resonant tunneling structures which include the effects of arbitrary potential profiles due to layered composition. ${ }^{13-15}$ Chen $^{16}$ models a one dimensional resonant tunneling diode using FEM and calculates a current-voltage curve based on a quantum hydrodynamic model. Electron wavefunctions and band structures for two dimensional quantum wires or quantum dots are analyzed using FEM by a number of investigators ${ }^{17-23}$. Tsuchida and Tsukada ${ }^{24}$ calculate the electronic structure of a perfect Si lattice using a three dimensional FEM formulation. However, none of the studies consider a three dimensional device, and none have considered the effects of strain on experimentally measured electronic properties of devices.

The finite element method is well suited for finding approximate solutions of boundary value problems for partial differential equations in finite domains, especially if the (unknown) exact solution is a minimizer of a total energy functional. Both the stress boundary value problem and the quantum mechanical boundary value problem in the present study are of this type. The central idea of the method is that an unknown continuous field in the domain is represented approximately in terms of its values at discrete points (nodes) within the domain; the goal is 
to determine optimal values for these nodal quantities. The domain is covered with areas (in two dimensions) or volumes (in three dimensions) whose boundaries are defined geometrically by the nodes; these areas or volumes are the elements. Fields are defined within each element in terms of the values of the nodal quantities on its boundary by means of a suitable interpolation scheme. With the complete field defined in terms of the nodal quantities, the total energy can be expressed in terms of the global vector of nodal quantities. Imposition of the condition that the actual values of the nodal quantities must render the total energy a minimum leads to a system of algebraic equations for these values. The method is ideally suited for numerical analysis by computer. In general, it is convergent as nodal spacing diminishes for elliptic partial differential equations which have unique solutions. It should be noted that the method is of far broader applicability than is implied by these introductory comments.

In this work, a finite element model is used to analyze both the continuum mechanics and the quantum mechanics of a strained semiconductor device. The strain field is shown to affect the performance of the device. The model is used to analyze devices studied experimentally by Akyüz et al. ${ }^{2}$ and Lukey et al. ${ }^{4}$, who show the effects of nonuniform strain on the resonant tunneling current vs. voltage characteristics of $\mathrm{Si}_{x} \mathrm{Ge}_{1-x}$ quantum wires and quantum dots, shown schematically in Figure 1. The main features of the model are described in Section II; these include the strain calculation, the treatment of the strain effects by deformation potential theory, and the quantum mechanical model. The finite element formulation for the two or three dimensional Schrödinger equation incorporating valence subband coupling and the nonuniform potential is outlined in Section III. The results of the calculations for the resonant tunneling structures are discussed in Section IV, and comparisons are made between the calculations and the available experimental results.

\section{CONTINUUM AND QUANTUM MECHANICAL MODELS}

The analysis of a strained semiconductor heterostructure is divided into three calculations. First, a linear elastic finite element calculation is made to determine the strain field, which is a function of the composition and the geometry of the structure. Second, the strain induced potential field is calculated using deformation potential theory. Third, the time-independent Schrödinger equation including the strain induced potential is solved numerically by means of the finite element method to obtain the spectrum of energies and wavefunctions of available states.

\section{A. Strain Field Calculation}

The strain field due to the constraint of epitaxy associated with the mismatched lattice parameters of the heterostructure layers is determined within the framework of linear elasticity theory. The structure is discretized spatially with a mesh which is more refined near free surfaces and in regions where the mismatch between adjacent layers is larger. The finite element mesh used for a strain calculation in the resonant tunneling diode quantum wire is shown in Figure 2. The mismatch condition is imposed in the finite element calculation by prescribing in each layer a uniform stressfree dilatation that is proportional to the bulk lattice parameter of the material in that layer. Continuity of displacements is required across the layer interfaces; it is this constraint which gives rise to stress. The outer surfaces are considered to be traction free, implying that certain 
components of the stress tensor vanish, and the material is allowed to relax until it reaches an equilibrium configuration. Thus, using a standard structural mechanics finite element program ${ }^{11}$, the complete state of stress, strain and displacement is determined throughout the device. Strain components are shown in Figures 3 and 4 for the resonant tunneling diode quantum wire. Strain is a tensor quantity, and its components show significant variation with position throughout the device. In smaller devices, the strain is more nonuniform due to the pervasive effect of relaxation at the free surfaces.

\section{B. Strain Induced Potential}

The components of strain induce a potential field that affects the wavefunctions and energies of the charge carriers in an otherwise perfect crystal. From first order perturbation theory, the strain induced potential that affects wavefunctions in subbands $\alpha$ and $\beta$ is formed from the tensor product $\left(\right.$ Singh $\left.^{25}\right)$

$$
V_{\epsilon}^{\alpha \beta}(\vec{r})=D_{i j}^{\alpha \beta} \epsilon_{i j}(\vec{r})
$$

where $\vec{r}$ is an arbitrary position vector, $\epsilon_{i j}(\vec{r})$ is the strain tensor field, and $D_{i j}^{\alpha \beta}$ is the deformation potential tensor for subbands $\alpha$ and $\beta$, which consists of components derived experimentally. The indices $i j$ range over the coordinate directions. For the $\mathrm{Si}_{x} \mathrm{Ge}_{1-x}$ material combination, the valence band electronic properties are dominated by the heavy hole and light hole subbands, so the $\alpha \beta$ basis includes the heavy hole $(\mathrm{HH})$ subbands denoted by $\mid \frac{3}{2}, \pm \frac{3}{2}>$, and the light hole (LH) subbands denoted by $\mid \frac{3}{2}, \pm \frac{1}{2}>$. The split-off subbands (SO) are ignored because the separation energy is considered to be large enough so that coupling effects can be neglected. The potential equation can be written as

$$
\begin{array}{cccc}
\mid \frac{3}{2},+\frac{3}{2}> & \mid \frac{3}{2},-\frac{3}{2}> & \mid \frac{3}{2},+\frac{1}{2}> & \mid \frac{3}{2},-\frac{1}{2}> \\
V_{\epsilon}^{\alpha \beta}(\vec{r})= \\
\mid \frac{3}{2},+\frac{3}{2}>\frac{3}{2},-\frac{3}{2}> \\
\mid \frac{3}{2},+\frac{1}{2}> \\
\mid \frac{3}{2},-\frac{1}{2}>
\end{array}\left(\begin{array}{cccc}
D_{i j}^{11}(\vec{r}) \epsilon_{i j}(\vec{r}) & D_{i j}^{12}(\vec{r}) \epsilon_{i j}(\vec{r}) & D_{i j}^{13}(\vec{r}) \epsilon_{i j}(\vec{r}) & D_{i j}^{14}(\vec{r}) \epsilon_{i j}(\vec{r}) \\
D_{i j}^{21}(\vec{r}) \epsilon_{i j}(\vec{r}) & D_{i j}^{22}(\vec{r}) \epsilon_{i j}(\vec{r}) & D_{i j}^{23}(\vec{r}) \epsilon_{i j}(\vec{r}) & D_{i j}^{24}(\vec{r}) \epsilon_{i j}(\vec{r}) \\
D_{i j}^{31}(\vec{r}) \epsilon_{i j}(\vec{r}) & D_{i j}^{32}(\vec{r}) \epsilon_{i j}(\vec{r}) & D_{i j}^{33}(\vec{r}) \epsilon_{i j}(\vec{r}) & D_{i j}^{34}(\vec{r}) \epsilon_{i j}(\vec{r}) \\
D_{i j}^{41}(\vec{r}) \epsilon_{i j}(\vec{r}) & D_{i j}^{42}(\vec{r}) \epsilon_{i j}(\vec{r}) & D_{i j}^{43}(\vec{r}) \epsilon_{i j}(\vec{r}) & D_{i j}^{44}(\vec{r}) \epsilon_{i j}(\vec{r})
\end{array}\right)
$$

where the terms $D_{i j}^{\alpha \beta}$ are the deformation potential tensors which range over the spatial dimensions $i$ and $j$. The deformation potential tensors contain material constants which vary spatially as the composition varies in the device; values of these constants for a wide range of materials are known from experiments. The repeated $i j$ indices indicate the scalar product (contraction over the product of second rank tensors) between the deformation potential tensor and the strain tensor. Details of the $D_{i j}^{\alpha \beta}$ terms are given in Appendix A, along with the material constants used in the calculations. Thus, for a calculated strain tensor function of position $\epsilon_{i j}(\vec{r})$, it is possible to calculate the deformation potential function of position $V_{\epsilon}^{\alpha \beta}(\vec{r})$ to be included in the quantum mechanical analysis.

\section{Quantum Mechanical Model}

In resonant tunneling structures, tunneling currents are determined by available quantized states for individual charge carriers. The energies and wavefunctions of a single carrier in the semicon- 
ductor structure are solutions of the time independent Schrödinger equation

$$
H_{k \cdot p}^{\alpha \beta}(\vec{r}) \Psi^{\beta}(\vec{r})+V^{\alpha \beta}(\vec{r}) \Psi^{\beta}(\vec{r})=E \Psi^{\alpha}(\vec{r})
$$

where $\Psi^{\alpha}(\vec{r})$ is the wavefunction in subband $\alpha, E$ is the energy, $H_{k \cdot p}^{\alpha \beta}(\vec{r})$ is the $\mathbf{k} \cdot \mathbf{p}$ Hamiltonian operator, and $V^{\alpha \beta}(\vec{r})$ is a potential function of position.

The $\mathbf{k} \cdot \mathbf{p}$ perturbation method is used to model the medium. Using this technique, there is a tensor function for the effective mass associated with each subband, and there are $\mathbf{k} \cdot \mathbf{p}$ terms coupling the effective masses in different subbands. Written in the same form as (2) the Hamiltonian is

$$
\begin{aligned}
& \left|\frac{3}{2},+\frac{3}{2}>\quad\right| \frac{3}{2},-\frac{3}{2}>\quad\left|\frac{3}{2},+\frac{1}{2}>\quad\right| \frac{3}{2},-\frac{1}{2}>
\end{aligned}
$$

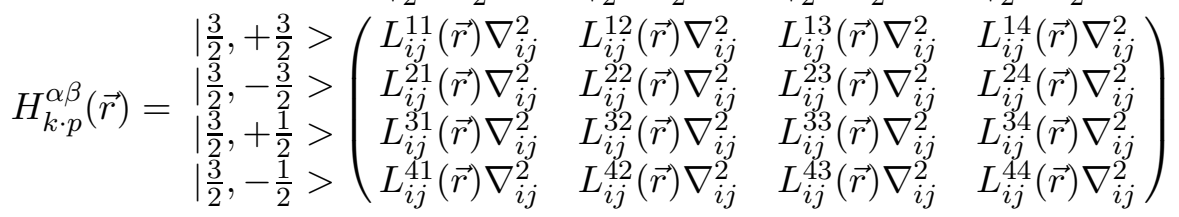

where the $L_{i j}^{\alpha \beta}$ tensors on the diagonal of the matrix are the effective mass tensors for each subband, and the off diagonal $L_{i j}^{\alpha \beta}$ tensors introduce a $\mathbf{k} \cdot \mathbf{p}$ coupling of subbands. Like the terms in the deformation potential tensors, the components of $L_{i j}^{\alpha \beta}$ contain material constants and thus vary spatially throughout the device. The exact forms of the $L_{i j}^{\alpha \beta}$ tensors, which come from the Luttinger-Kohn Hamiltonian, are given in Appendix A.

The deformation potential and effective mass material constants in (2) and (4) are functions of the local composition of the device. Values for the constants in each layer are given by linear interpolation of the material constants associated with each of the pure elements composing that layer.

The nonuniform potential $V^{\alpha \beta}(\vec{r})$ consists of contributions from two sources and is given by

$$
V^{\alpha \beta}(\vec{r})=V_{c}^{\alpha \beta}(\vec{r})+V_{\epsilon}^{\alpha \beta}(\vec{r})
$$

where $V_{c}^{\alpha \beta}(\vec{r})$ is due to the valence band alignment of material at a given position in the device, and $V_{\epsilon}^{\alpha \beta}(\vec{r})$ is the strain induced potential given in (1). The strain induced potential $V_{\epsilon}^{\alpha \beta}(\vec{r})$, like the components of the strain tensor, is in general nonuniform in both the lateral directions and the vertical direction in the structure. The composition based band offset potential $V_{c}^{\alpha \beta}(\vec{r})$ is nonuniform only in the growth, or vertical direction in the structure. The total potential $V^{\alpha \beta}(\vec{r})$ is shown in Figure 5 for a representative quantum dot calculation.

\section{FINITE ELEMENT TECHNIQUE FOR THE SCHRÖDINGER EQUATION}

\section{A. Finite Element Formulation}

The physical domain of the device is discretized into a mesh of nodes and elements. Elements used for the quantum wires and quantum dots are described in Appendix B. The mesh extends to the free or insulated surfaces, which impose an infinite potential on the wavefunction. The 
wavefunctions and energies of the states localized in the active region are insensitive to remote boundary conditions, i.e. conditions at boundaries located a large distance away relative to the active region size. The mesh is more refined in the active region of the device, where large wavefunction gradients are expected.

The form of the Schrödinger equation to be solved on the finite element mesh is obtained by minimizing the total variation of the weak form of the equation with respect to the wavefunction. The minimum in variation with respect to the wavefunction corresponds physically to a minimum energy. Details of the complete variational formulation of the finite element technique are included in Appendix B. The functional corresponding to the weak form of the time independent Schrödinger equation with a nonuniform potential (3) is given by

$$
\Pi\left(\Psi^{\alpha}\right)=\int_{R} \nabla \Psi^{\alpha} L^{\alpha \beta} \nabla \Psi^{\beta} d R+\int_{R} \Psi^{\alpha} V^{\alpha \beta} \Psi^{\beta} d R-E \int_{R} \Psi^{\alpha} \Psi^{\beta} d R
$$

where $\Psi^{\alpha}, L^{\alpha \beta}$, and $V^{\alpha \beta}$ are functions of position in the structure. The term $L^{\alpha \beta}$ is taken to be constant within each element of the mesh. The fields $\Psi^{\alpha}, \nabla \Psi^{\alpha}$, and $V^{\alpha \beta}$ are represented by their nodal values. Values throughout each element are determined by interpolation according to the particular shape functions that are adopted. Thus, for shape functions $N(\vec{r})$ used here, these fields are written as

$$
\Psi^{\alpha}=\sum_{A=1}^{\text {all nodes }} \Psi_{A}^{\alpha} N_{A}(\vec{r}) \quad \nabla \Psi^{\alpha}=\sum_{B=1}^{\text {all nodes }} \Psi_{B}^{\alpha} \nabla N_{B}(\vec{r}) \quad V^{\alpha \beta}=\sum_{C=1}^{\text {all nodes }} V_{C}^{\alpha \beta} N_{C}(\vec{r})
$$

where the coefficients in the summations are the values of the fields at the individual nodes. The functional $\Pi\left(\Psi^{\alpha}\right)$ is rewritten in a discrete form using (7) and then minimized with respect to the nodal values of the wavefunction, $\Psi_{A}^{\alpha}$. Integrals over the region $R$ of the entire structure are replaced with integrals over individual element volumes $\left(\Omega^{e}\right)$ and a summation over all elements, so that the finite element form of the Schrödinger equation becomes

$$
\sum^{\text {elems }}\left[\sum_{A} \sum_{B} \int_{\Omega^{e}}\left(\nabla N_{A} L^{\alpha \beta} \nabla N_{B}+N_{A} N_{B} \sum_{C} V_{C}^{\alpha \beta} N_{C}\right) d \Omega\right] \Psi_{B}^{\beta}=E \sum^{\text {elems }}\left[\sum_{A} \sum_{B} \int_{\Omega^{e}} N_{A} N_{B} d \Omega\right] \Psi_{B}^{\alpha}
$$

which is a form ideally suited for computation.

This form of the Schrödinger equation can be much more simply expressed as

$$
K_{i j} \Psi_{j}=E M_{i j} \Psi_{j}
$$

where the indices $i$ and $j$ range over all nodal wavefunction degrees of freedom, and the repeated indices indicate a summation. Details of the assembly of the matrices $K$ and $M$ are given in Appendix B.

\section{B. Finite Element Solution}

\section{Energies and wavefunctions}

The finite element expression of the Schrödinger equation (9) is in the form of a generalized eigenvalue equation. The problem has $n \alpha$ solutions, where $n$ is the total number of nodes in the 
mesh and $\alpha$ is the number of subbands in the quantum mechanical basis. The solutions consist of energies $E$ and wavefunctions $\Psi$, of which the lowest energy states are the most accurate.

Of the $n \alpha$ eigenstates, some states can be found for which the wavefunction $\Psi(\vec{r})$ or the probability density $|\Psi(\vec{r})|^{2}$ is confined to the active region of the device. Examples of eigenstates confined to the quantum well layers of the quantum dot are shown in Figure 6. The lowest energy states of these confined eigenstates are the most relevant to transport. For the example of the resonant tunneling diode, sequential tunneling through the double barriers is possible only when the tunneling carriers have energies equal to the energies of the confined states in the quantum well. Thus, over a range of applied biases, the excited carriers can access the confined states and induce a tunneling current only at certain resonances corresponding to the spectrum of eigenvalues given by the finite element solution.

The energy and wavefunction solutions reflect the effects of strain, composition, and effective mass on the carriers. The valence band offset in adjacent layers imposes a large relative potential on the charge carriers which results in confinement to the quantum well region of the device. The strain induced potential is considerably smaller than the valence band potential, but it also shifts the wavefunctions spatially and energetically. Confined states corresponding to valence subbands with higher effective masses occur at lower energies.

\section{Density of states}

The density, with respect to energy, of states confined to the active region of the device can be obtained directly from the spectrum of eigenstates given in the finite element solution. This density of confined states is a real space measure of the electronic properties of the device. Effects due to strain, composition, size, and device characteristics can be seen in the density of confined states. For the example of the resonant tunneling diode, the density of confined states can be used to examine strain effects on the resonant tunneling spectrum. A larger density of confined states with a given energy increases the probability of resonant tunneling by carriers with the same energy. A high probability of tunneling at a given energy is measured experimentally as a tunneling current peak. Thus, the density of confined states can be used to make contact with experimental data.

A Gaussian broadening technique can be used to calculate a continuous density of confined states function $\rho(E)$. The energy $E_{i}$ of each confined state is broadened by a narrow, normalized Gaussian distribution, and a summation over all $n$ states gives $\rho(E)$ as

$$
\rho(E)=\sum_{i=1}^{n} \frac{1}{2 a \sqrt{\pi}} \exp \left(-\left(E-E_{i}\right)^{2} / 4 a^{2}\right)
$$

where $a$ is a free parameter that controls the width of the Gaussian distributions and thus the smoothness of the density of states $\rho(E)$. The parameter $a$ is chosen to bring out the general features of $\rho(E)$; the broadening of each state is larger than the typical separation of individual eigenstates, but narrow enough to bring out features of the density of states that are due to small groups of related eigenstates. Typical values of $a$ are on the order of $1 \mathrm{meV}$. 


\section{RESULTS AND COMPARISON TO EXPERIMENTS}

\section{A. The Quantum Wire}

\section{Physical system}

The quantum wire considered here is a long, row-shaped, layered structure of fixed total height $h$. The geometry of the structure and the thickness and composition of each layer, based on the experimental work of Lukey et al. ${ }^{4}$, are shown in Figure 7 . The middle layers of the device are considered to be the active region, and include the quantum well layer $\left(\mathrm{Si}_{0.78} \mathrm{Ge}_{0.22}\right)$ and the two undoped barrier layers $(\mathrm{Si})$. Surrounding the active region are strained $\left(\mathrm{Si}_{0.78} \mathrm{Ge}_{0.22}\right)$ emitter and collector regions; the thick outermost layers of the device are Si. A range of widths $w$ are considered in order to model size-dependent strain effects and to compare results with experimental data.

The device operates by the sequential tunneling of charge carriers from the doped region above the barrier layer, into the quantum well layer, and then through the lower barrier layer. Resonant tunneling spectroscopy is done experimentally by applying a bias across the device and measuring the current of tunneling carriers that is induced. A resonant tunneling spectrum can be compiled by measuring the induced currents associated with a range of applied biases. The experimental result is a resonant tunneling $\mathrm{I}(\mathrm{V})$ curve.

\section{Strain field}

The strain and displacement fields for this geometry are two dimensional since the constraining effect of the material in the direction along the long axis of the wire imposes a state of plane strain. The mesh is more refined in the active region of the structure and near the traction free lateral surfaces, where the deformation is expected to be more nonuniform. The $\epsilon_{22}$ component of strain (extensional strain in the $x_{2}$ direction) is shown in Figure 3 in and near the active region of the device, plotted on a deformed mesh that shows the actual displacements, magnified for clarity. In Figure 4 the $\epsilon_{11}$ component of strain along the centerline of the quantum well layer is plotted for structures with three different widths. The important features are that the strain is a tensor valued function and that the relaxing effect of the free surface and the multilayered composition of the structure lead to nonuniform strain components.

\section{Results of the quantum mechanical calculation}

Solution of the quantum mechanical problem gives the energies and wavefunctions of states confined to the quantum well layer in the wire. From this spectrum of states, a density of confined states is calculated. The density of confined states for a narrow wire $(\mathrm{w}=250 \mathrm{~nm})$ is shown in Figure 8, representative eigenstates are shown in Figures 9 and 10, and the density of confined states for wide and narrow wires are compared in Figure 11.

The results of the calculations are consistent with some experimental observations of Lukey et al. . First, the strain separates the resonant current peaks associated with the heavy hole and light hole subbands, as shown in Figure 8. The calculation gives a peak separation of about 20 $\mathrm{meV}$, which would correspond to a bias shift of roughly $50 \mathrm{mV}$. The experimentally measured separation is approximately $90 \mathrm{mV}$. However, it is important to note that the calculation shown 
is for a wire aligned along a (100) crystalline axis. The experiments of Lukey et al. measured wires aligned along a (110) axis, which would exhibit more sensitivity to strain in the electronic properties due to the form of the deformation potential tensor $D_{i j}^{\alpha \beta}$, and thus presumably a wider strain induced peak separation. The second characteristic evident in Figure 8 is the presence of additional fine features in the density of states. Fine structure is also observed experimentally in the $\mathrm{w}=250 \mathrm{~nm}$ device. An examination of the states present over a range of energies shows that the fine features in the density of states are due to groups of similar states separated in energy by the influence of nonuniform strain, as shown in Figures 9 and 10.

The size dependence of the strain effect is demonstrated in the densities of confined states for wires with widths of $250 \mathrm{~nm}$ and $900 \mathrm{~nm}$ in Figure 11. The main feature is the increase in the energy separation of the heavy hole and light hole peaks as the wire width increases. This is due to the reduced effect of free surface strain relaxation in larger devices, where the average strain values approach the bulk film mismatch strains. In the smallest devices, the strain is relaxed over a significant portion of the volume, so the average strain is smaller and the energy separation between the heavy hole and light hole peaks is smaller.

\section{B. The Quantum Dot}

\section{Physical system}

The quantum dot considered here is cylindrical in shape and of fixed height. The features of this structure are based on the experimental work of Zaslavsky et al. ${ }^{1}$ and Akyüz et al. ${ }^{2}$ and are shown in Figure 12 for a representative calculation. The three middle layers, which consist of the quantum well $\left(\mathrm{Si}_{0.75} \mathrm{Ge}_{0.25}\right)$ and the barriers $(\mathrm{Si})$, make up the active region of the device. Surrounding the active region are the emitter and collector regions $\left(\mathrm{Si}_{0.75} \mathrm{Ge}_{0.25}\right)$ which have relatively low strain due to the outermost layers which are graded in composition. A range of cylinder diameters $d$ is considered.

The quantum dot device operates on the same resonant tunneling principle as the quantum wire. Carriers tunnel sequentially from the emitter region, through the upper barrier into states in the quantum well, and then through the lower barrier into the collector region. The experimental $\mathrm{I}(\mathrm{V})$ curve is a measure of the resonant tunneling spectrum.

\section{Strain field}

The stress, strain, and displacement fields are axisymmetric for this geometry. The mesh used to calculate the strain extends from the center axis of the structure outward, with increasing refinement near the outer, traction free surface of the device, where the fields are expected to be more nonuniform. Figure 13 shows the $\epsilon_{22}$ component of strain (extensional strain in the axial direction) on a deformed mesh, and Figure 14 shows $\epsilon_{11}$, the extensional strain along a radial line in the midplane of the quantum well. As in the case of the quantum wire, the strain is very nonuniform, and the extent of the nonuniformity increases in smaller structures. This is due to the more significant effect of free surface proximity. 


\section{Results of the quantum mechanical calculation}

For the three-dimensional quantum dot, a reduced quantum mechanical basis is adopted in order to limit the total number of degrees of freedom in the calculation. To model only the lowest energy heavy hole states, it is sufficient to consider a one-dimensional quantum mechanical basis where only the $\mid \frac{3}{2},+\frac{3}{2}>$ band is examined, but it is still possible to consider an anisotropic effective mass.

Two significant results compare favorably to the experiments of Zaslavksy et al. ${ }^{1}$ and Akyüz et al. ${ }^{2}$. First, as seen in Figure 15, there is a size dependence of the strain effect on the resonant energies that is similar to the effect in the quantum wires. In larger diameter devices, the average strain is higher because free surface relaxation is less significant, so the peak is shifted further from an idealized case without strain.

Second, in the smallest devices, there are additional fine features in the density of states that are shown in Figure 16 to be the result of the influence of nonuniform strain. In agreement with the suggestion of Akyüz et al. ${ }^{2}$, it is evident that the relaxed strain near the lateral surface leads to low energy ring-like heavy hole states. The strain induced energy shifting of groups of states produces features in the density of confined states that are consistent with the $\mathrm{I}(\mathrm{V})$ curves for devices of the same size. Figure 17 shows a calculated density of confined states plot and a measured $\mathrm{I}(\mathrm{V})$ curve for a $\mathrm{d}=250 \mathrm{~nm}$ quantum dot with $10 \mathrm{meV}$ on the energy axis equal to $25 \mathrm{mV}$ on the bias axis. Many of the features of the $\mathrm{I}(\mathrm{V})$ curve are predicted qualitatively in the density of confined states curve, including fine structure below and above the main heavy hole resonance energy. However, the density of confined states is not equivalent to the resonant tunneling current; the calculation does not consider some important physical effects, most notably the roughly linear background current in the I(V) relationship.

\section{CONCLUSIONS}

A finite element technique is presented here which allows for the calculation of strain effects on the electronic and transport properties of strained quantum wires and dots. The approach is similar to some recent work as it is based on a simplified quantum mechanical model ${ }^{5-8}$, but the flexibility of meshing and the low computational cost of the finite element method offer easy access to results which can be compared to experimental data.

The technique is used to examine mismatch strain effects in quantum wires and quantum dots that operate on a simple single carrier sequential tunneling effect. Strain effects are shown to explain several reported trends in experimental data. In particular, two features of the mismatch strain in the devices have strong effects on the calculated electronic and transport properties. First, the average effect of the strain is to separate the resonant energy peaks associated with the individual valence subbands in the material. In larger devices, the strain is less relaxed by the free surfaces, so the HH-LH subband energy separation is larger. Second, strain nonuniformity in the devices is responsible for fine structure in the resonant tunneling current peaks. This effect is the source of low energy ring-like states that are found in the small cylindrical quantum dots, and the edge states found in quantum wires over a range of sizes.

The main weaknesses in the method are in the simplified quantum mechanical model. The real-space calculation is based on a $k$-space material model that is accurate near $k=0$. The 
resonant tunneling model assumes ballistic transport of a single charge carrier, and contact is made with experiments in only an approximate way. A linear elastic constitutive model provides a good approximation for the strain, although the approach has limitations for such small, highly strained structures $^{25}$. Finally, the strain effect is treated as a linear perturbation to a perfect crystal Hamiltonian, so the fully coupled nature of strain and electronic properties through the chemical bonding is not considered. However, the technique shown here is a promising, computationally inexpensive way to determine strain effects on electronic properties in semiconductors. The means to overcome the noted shortcomings are under development.

\section{ACKNOWLEDGEMENTS}

One of the authors (HTJ) would like to acknowledge helpful discussions with V. B. Shenoy and R. Phillips regarding the application of the finite element method to quantum mechanics. The authors are also grateful for the cooperation of J. Caro in providing access to experimental data. The research support of the Office of Naval Research, Contract N00014-95-1-0239, and the MRSEC Program of the National Science Foundation, under Award DMR-9632524, is gratefully acknowledged. 


\section{REFERENCES}

1 A. Zaslavsky, K. R. Milkove, Y. H. Lee, B. Ferland, and T. O. Sedgewick, Appl. Phys. Lett. 67, 3921 (1995).

2 C. D. Akyüz, A. Zaslavsky, L. B. Freund, D. A. Syphers, and T. O. Sedgewick, Appl. Phys. Lett. 72, 1739 (1998).

3 P. Gassot, U. Gennser, D. M. Symons, A. Zaslavsky, and D. A. Gruztmacher, J. C. Portal, submitted to Phys. Rev. B (1997).

${ }^{4}$ P. W. Lukey, J. Caro, T. Zijlstra, E. van der Drift, and S. Radelaar, Phys. Rev. B 57, 7132 (1998).

${ }^{5}$ C. Pryor, M-E. Pistol, L. Samuelson, Phys. Rev. B 56, 10404 (1997).

${ }^{6}$ C. Pryor, Phys. Rev. B 57, 7190 (1998).

7 C. Pryor, http://xxx.lanl.gov/abs/cond-mat/9801225.

8 A. J. Williamson, A. Zunger, A. Canning, http://xxx.lanl.gov/abs/cond-mat/9801191.

9 A. Zunger, MRS Bull. 23 15, 1998.

10 T. J. Gosling and L. B. Freund, Acta mater. 44, 1 (1996).

11 ABAQUS, Version 5.5, Hibbitt, Karlsson \& Sorensen, Inc., Pawtucket, RI 02860, U. S. A. (1995).

12 J. Linderberg, Comp. Phys. Reports 6, 209 (1987).

${ }^{13}$ K. Nakamura, A. Shimizu, M. Koshiba, and K. Hayata, IEEE J. Quantum Electron. 25, 889 (1989).

14 A. Zhao, S. R. Cvetkovic, and Z. A. Yang, Opt. Quant. Electron. 25, 845 (1993).

15 T. L. Li and K. J. Kuhn, J. Comp. Phys. 115, 288 (1994).

16 Chen, Computers Math. Applic. 31, 17 (1996).

${ }^{17}$ K. Kujima, K. Mitsunaga, and K. Kyuma, Appl. Phys. Lett. 55, 882, (1989)

18 D. J. Kirkner, C. S. Lent, and S. Sivaprakasm, I. J. Num. Meth. Eng. 29, 1527, (1990)

19 Z. Wu and P. P. Ruden, J. Appl. Phys. 74, 6234 (1993).

20 Y. Wang, J. Wang, and H. Guo, Phys. Rev. B 49, 1928 (1994).

21 T. Inoshita and H. Sakaki, J. Appl. Phys. 79, 269 (1996).

22 J. C. Yi and N. Dagli, IEEE J. Quantum Electron. 31, 208 (1995).

23 T. Inoshita and H. Sakaki, J. Appl. Phys. 79, 269 (1996).

${ }^{24}$ E. Tsuchida and M. Tsukada, Phys. Rev. B 52, 5573 (1995).

25 J. Singh, Physics of Semiconductors and Their Heterostructures, (McGraw-Hill, New York, 1993), p. 228.

${ }^{26}$ C. Pryor, J. Kim, L. W. Wang, A. Williamson and A. Zunger, J. Appl Phys. 83, 51998. 


\section{Appendix A}

The strain induced potential $V_{\epsilon}^{\alpha \beta}(\vec{r})$ is given by

$$
\begin{aligned}
& \left.\left|\frac{3}{2},+\frac{3}{2}>\quad\right| \frac{3}{2},-\frac{3}{2}>\quad\left|\frac{3}{2},+\frac{1}{2}>\quad\right| \frac{3}{2},-\frac{1}{2}\right\rangle
\end{aligned}
$$

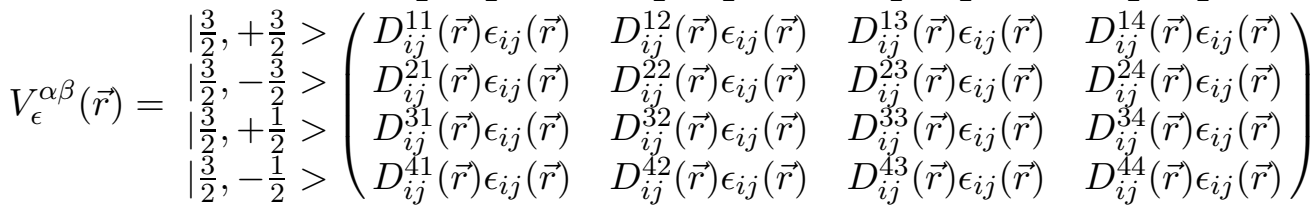

where each component $D_{i j}^{\alpha \beta}(\vec{r})$ of the matrix for fixed $\alpha \beta$ forms a scalar product with the strain tensor $\epsilon_{i j}(\vec{r})$ through summation over $i$ and $j$. And similarly, the $\mathbf{k} \cdot \mathbf{p}$ Hamiltonian given by Luttinger and Kohn takes the form

$$
\begin{aligned}
& \left|\frac{3}{2},+\frac{3}{2}>\quad\right| \frac{3}{2},-\frac{3}{2}>\quad\left|\frac{3}{2},+\frac{1}{2}>\quad\right| \frac{3}{2},-\frac{1}{2}>
\end{aligned}
$$

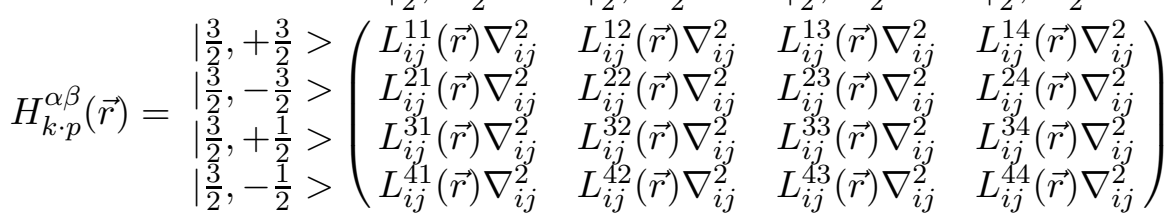

where the each of the matrix components $L_{i j}^{\alpha \beta}(\vec{r})$ for fixed $\alpha \beta$ form a scalar product with the operator $\nabla_{i j}^{2}$. The components $D_{i j}^{\alpha \beta}(\vec{r})$ and $L_{i j}^{\alpha \beta}(\vec{r})$ have very similar form. The deformation potential components $D_{i j}^{\alpha \beta}(\vec{r})$ are

$$
\begin{gathered}
D_{i j}^{11}(\vec{r})=D_{i j}^{22}(\vec{r})=\left[\begin{array}{ccc}
a+\frac{b}{2} & 0 & 0 \\
0 & a+\frac{b}{2} & 0 \\
0 & 0 & a-b
\end{array}\right] \\
D_{i j}^{33}(\vec{r})=D_{i j}^{44}(\vec{r})=\left[\begin{array}{ccc}
a-\frac{b}{2} & 0 & 0 \\
0 & a-\frac{b}{2} & 0 \\
0 & 0 & a+b
\end{array}\right] \\
D_{i j}^{13}(\vec{r})=D_{i j}^{31^{*}}(\vec{r})=-D_{i j}^{24^{*}}(\vec{r})=-D_{i j}^{42}(\vec{r})=\left[\begin{array}{ccc}
0 & 0 & -i \frac{d}{2} \\
0 & 0 & -\frac{d}{2} \\
-i \frac{d}{2} & -\frac{d}{2} & 0
\end{array}\right] \\
D_{i j}^{14}(\vec{r})=D_{i j}^{23^{*}}(\vec{r})=D_{i j}^{32}(\vec{r})=D_{i j}^{41 *}(\vec{r})=\left[\begin{array}{ccc}
\frac{\sqrt{3}}{2} b & -i \frac{d}{2} & 0 \\
-i \frac{d}{2} & -\frac{\sqrt{3}}{2} b & 0 \\
0 & 0 & 0
\end{array}\right] \\
D_{i j}^{12}(\vec{r})=D_{i j}^{21}(\vec{r})=D_{i j}^{34}(\vec{r})=D_{i j}^{43}(\vec{r})=\left[\begin{array}{ccc}
0 & 0 & 0 \\
0 & 0 & 0 \\
0 & 0 & 0
\end{array}\right]
\end{gathered}
$$

The Hamiltonian components $L_{i j}^{\alpha \beta}(\vec{r})$ can be obtained by making the substitutions $\frac{\hbar^{2}}{2 m_{0}} \gamma_{1} \leftrightarrow a$, $\frac{\hbar^{2}}{m_{0}} \gamma_{2} \leftrightarrow b, \frac{\sqrt{3} \hbar^{2}}{m_{0}} \gamma_{3} \leftrightarrow d$ into the expressions for the components $D_{i j}^{\alpha \beta}(\vec{r})$, where $\gamma_{1}, \gamma_{2}, \gamma_{3}$ are the 
Luttinger-Kohn parameters. Values for the deformation potential constants and the LuttingerKohn parameters for $\mathrm{Si}$ and Ge are given below. Values for alloys of $\mathrm{Si}$ and Ge are interpolated from values for the bulk materials.

\begin{tabular}{|l||l|l|l|l|l|l|}
\hline & $a(e V)$ & $b(e V)$ & $d(e V)$ & $\gamma_{1}$ & $\gamma_{2}$ & $\gamma_{3}$ \\
\hline$S i$ & 2.1 & -1.5 & -3.4 & 4.29 & 0.34 & 1.45 \\
\hline$G e$ & 2.0 & -2.2 & -4.4 & 13.4 & 4.24 & 5.59 \\
\hline
\end{tabular}

\section{Appendix B}

To obtain the finite element form of the Schrödinger equation, the physical region is divided into elements, which are taken here to be 4-noded quadrilaterals for the two dimensional quantum wire and 8-noded bricks for the three dimensional quantum dot. The wavefunction $\Psi^{\alpha}$, wavefunction gradient $\nabla \Psi^{\alpha}$, and potential $V^{\alpha \beta}$ are expressed in terms of discretized values at the nodes, and values within the elements are determined by linear interpolation using linear shape functions $N(\vec{r})$. A group of quadrilateral elements and the linear shape function for an associated node is shown in Figure 18.

The form of the Schrödinger equation to be solved is

$$
L^{\alpha \beta}(\vec{r}) \nabla^{2} \Psi^{\beta}(\vec{r})+V^{\alpha \beta}(\vec{r}) \Psi^{\beta}(\vec{r})=E \Psi^{\alpha}(\vec{r})
$$

The weak form of the equation is obtained by multiplying by $\Psi(\vec{r})$ and integrating over the volume of the body. The first term is integrated by parts, and the functional corresponding to the weak form is given by

$$
\Pi\left(\Psi^{\alpha}\right)=\int_{R} \nabla \Psi^{\alpha} L^{\alpha \beta} \nabla \Psi^{\beta} d R+\int_{R} \Psi^{\alpha} V^{\alpha \beta} \Psi^{\beta} d R-E \int_{R} \Psi^{\alpha} \Psi^{\beta} d R
$$

The spatially varying fields are then discretized using the shape functions $N(\vec{r})$ to get

$\Pi\left(\Psi_{A}^{\alpha}\right)=\sum_{A} \sum_{B}\left[\int_{R} \Psi_{A}^{\alpha} \nabla N_{A} L^{\alpha \beta} \Psi_{B}^{\beta} \nabla N_{B} d R+\int_{R} \Psi_{A}^{\alpha} N_{A} \sum_{C} V_{C}^{\alpha \beta} N_{C} \Psi_{B}^{\beta} N_{B} d R-E \int_{R} \Psi_{A}^{\alpha} \Psi_{B}^{\beta} N_{A} N_{B}\right] d R$

The total variation of the functional $\Pi\left(\Psi_{A}^{\alpha}\right)$ is then minimized with respect to the nodal values of the wavefunction $\Psi_{B}^{\beta}$ so that

$$
\frac{d \Pi\left(\Psi_{A}^{\alpha}\right)}{d \Psi_{B}^{\beta}}=0
$$

thus

$$
\Psi_{A}^{\alpha} \sum_{A} \sum_{B}\left[\int_{R} \nabla N_{A} L^{\alpha \beta} \nabla N_{B} d R+\int_{R} N_{A} \sum_{C} V_{C}^{\alpha \beta} N_{C} N_{B} d R-E \int_{R} N_{A} N_{B} d R\right]=0
$$

Replacing integrals over the region $R$ with integration over individual element volumes $\left(\Omega^{e}\right)$ and a summation over all elements, the final finite element form of the equation becomes

$\sum^{\text {elems }}\left[\sum_{A} \sum_{B} \int_{\Omega^{e}}\left(\nabla N_{A} L^{\alpha \beta} \nabla N_{B}+N_{A} N_{B} \sum_{C} V_{C}^{\alpha \beta} N_{C}\right) d \Omega\right] \Psi_{B}^{\beta}=E \sum^{\text {elems }}\left[\sum_{A} \sum_{B} \int_{\Omega^{e}} N_{A} N_{B} d \Omega\right] \Psi_{B}^{\alpha}$ 
The contribution of a single element to the left hand side of the equation is given by the element stiffness matrix. The integration over the element is done numerically at a set of quadrature points. The construction of an element stiffness matrix for the case of two spatial dimensions and a four subband quantum mechanical basis is as follows:

$$
\begin{aligned}
& k_{\alpha \beta}^{e}=\int_{\Omega^{e}}\left(\nabla N_{A} L^{\alpha \beta} \nabla N_{B}+N_{A} N_{B} \sum_{C} V_{C}^{\alpha \beta} N_{C}\right) d \Omega \\
& =\sum_{l=1}^{\text {int.pts. }} \underbrace{\nabla N_{A}}_{16 \times 16} \underbrace{L^{\alpha \beta}}_{8 \times 8} \underbrace{\nabla N_{B}}_{8 \times 16}+\underbrace{\underbrace{N_{A}}_{4 \times 4} \underbrace{\sum^{\text {nodes }}\left(V_{C}^{\alpha \beta} N_{C}\right)}_{16 \times 16} \underbrace{N_{B}}_{4 \times 16}]_{l}}_{16 \times 4}
\end{aligned}
$$

where the tensors $D_{i j}^{\alpha \beta}$ and $L_{i j}^{\alpha \beta}$ given in Appendix 1 reduce to $2 \times 2$ matrices, and the shape function matrix is given by

$$
N_{A}=\left[\begin{array}{cccc|cccccccccccc}
N_{1} & N_{2} & N_{3} & N_{4} & 0 & \ldots & \ldots & 0 & \ldots & \ldots & 0 & \ldots & \ldots & 0 & \ldots & \ldots \\
\hline 0 & \ldots & \ldots & 0 & N_{1} & N_{2} & N_{3} & N_{4} & 0 & \ldots & \ldots & 0 & \ldots & \ldots & 0 & \ldots \\
\ldots & 0 & \ldots & \ldots & 0 & \ldots & \ldots & 0 & N_{1} & N_{2} & N_{3} & N_{4} & 0 & \ldots & \ldots & 0 \\
\ldots & \ldots & 0 & \ldots & \ldots & 0 & \ldots & \ldots & 0 & \ldots & \ldots & 0 & N_{1} & N_{2} & N_{3} & N_{4}
\end{array}\right]
$$

and the shape function derivative matrix $\nabla N_{A}$ follows in the same form.

The contribution of a single element to the right hand side of equation (B6) is referred to as the element mass matrix and is constructed in a similar way as $k_{\alpha \beta}^{e}$. The final finite element matrix form of the Schrödinger equation, given by

$$
K_{i j} \Psi_{j}=E M_{i j} \Psi_{j}
$$

is constructed by assembling the element stiffness matrices and element mass matrices into global element and mass matrices, $K_{i j}$ and $M_{i j}$, for the entire device. 


\section{List of Captions}

1. Schematics of the quantum wire and quantum dot. The row-shaped quantum wire (Lukey et al. ) has width $\mathrm{w}$ and extends a distance much larger than $\mathrm{w}$ in the (010) direction. The cylindrical quantum dot (Akyüz et al. ) has diameter d.

2. Finite element mesh used to calculate strain in the quantum wire. Since the strain is symmetric laterally and vertically about the center axes of the device, it is possible to model one quarter of the cross section of the structure only. A portion of the highly refined mesh near the edge of the active region is shown.

3. Direct vertical component of strain in the upper half of the quantum wire, from the center to the edge. Displacements of the free outer surface of the device can be seen at the right edge of the plot. The layers, from the bottom, represent the well and barrier layers (highly nonuniformly strained) and the emitter and topmost Si layers.

4. Direct lateral component of strain in the center of the well layer of the quantum wire. The strain is uniform near the center of the wire (left) but highly nonuniform near the edge (right). In larger wires the strain is less relaxed and less nonuniform.

5. The total potential $V(r)$ for the heavy hole band in the strained quantum dot. The potential in the barrier layers is high relative to the potential in the quantum well layer; the potential is axisymmetric, and radially nonuniform throughout the device.

6. Representative probability densities in a $d=50 \mathrm{~nm}$ quantum dot. On the left is a low energy state with six-fold angular quantization localized in a ring-like region near the outer edge of the active region. On the right is a higher energy state, localized in the center of the active region, with two fold angular quantization and two fold quantization in the $\mathrm{z}$ direction.

7. Schematic of the quantum wire geometry and composition in the active region. The $59 \AA$ Si barriers surround the $33 \AA \mathrm{Si}_{0.78} \mathrm{Ge}_{0.22}$ quantum well layer. An applied bias $\mathrm{V}$ induces a tunneling current in the $\mathrm{z}$ direction.

8. Density of confined states in the strained quantum wire of width $250 \mathrm{~nm}$. The two large peaks in the dashed curve show the calculated heavy hole and light hole resonances without considering strain effects. Strain causes the resonances to separate in energy and induces fine structure in the density of confined states. States at points A (edge state) and B (light hole state) are shown in Figures 9 and 10.

9. Probability density for a lower energy confined state. The four cross sections of the active region show the probability density associated with each of the four valence subbands for a given eigenstate. The wavefunction in this eigenstate is of mixed type, and localized near the edge of the structure due to the free surface strain relaxation.

10. Probability density for a higher energy confined state. The predominantly $\mid \frac{3}{2},+\frac{1}{2}>$ type wavefunction is localized in the center of the quantum well, but shows some effects of the nonuniform potential near the lateral surfaces and strong mixing with the $\mid \frac{3}{2},-\frac{3}{2}>$ subband. 
11. Densities of confined states for two quantum wires of different widths. The higher average strain in the wider device results in a larger energy separation between heavy hole and light hole resonance peaks. The heavy hole peak is shown to be shifted by the strain more than the light hole peak.

12. Geometry and composition of the quantum dot device (Akyüz et al. ). The $45 \AA$ Si barriers surround the $35 \AA \mathrm{Si}_{0.75} \mathrm{Ge}_{0.25}$ well. The total height is $80 \mathrm{~nm}$, and the diameter $\mathrm{d}$ varies. An applied bias $\mathrm{V}$ induces a tunneling current in the $\mathrm{z}$ direction.

13. Vertical component of strain in the upper half of the quantum dot, from the center to the edge. The plot is deformed to show the displacement in the structure, and the strain is highly nonuniform, particularly in the active region of the device. The two layers at the bottom are the quantum well and top barrier layers.

14. Direct lateral component of strain in the well layer of the quantum dot, from the center to the edge. The strain is more relaxed and more nonuniform in the smaller structures. The strain profile is very similar to the profile in the quantum wire.

15. Densities of heavy hole confined states for a range of quantum dot diameters. The variation in average strain levels results in the shifting of the peaks for dots of different sizes. The energy shift corresponds to the bias shift measured by Zaslavsky et al. .

16. The effect of strain induced lateral confinement on the density of confined states. The features of the density of confined states are due to groups of eigenstates with similar lateral quantization.

17. Fine structure in the density of confined states in the $d=250 \mathrm{~nm}$ quantum dot (Akyüz et al. ). The calculated density of confined states is consistent with the experimental currentvoltage curve. The density of confined states calculation does not account for the roughly linear background current that is observed experimentally.

18. Elements, nodes, and a representative shape function in the two dimensional finite element formulation. 
This figure "fig01.JPG" is available in "JPG" format from: http://arxiv.org/ps/cond-mat/9806029v1 
This figure "fig02.JPG" is available in "JPG" format from: http://arxiv.org/ps/cond-mat/9806029v1 
This figure "fig03.JPG" is available in "JPG" format from: http://arxiv.org/ps/cond-mat/9806029v1 
This figure "fig04.JPG" is available in "JPG" format from: http://arxiv.org/ps/cond-mat/9806029v1 
This figure "fig05.JPG" is available in "JPG" format from: http://arxiv.org/ps/cond-mat/9806029v1 
This figure "fig06.JPG" is available in "JPG" format from: http://arxiv.org/ps/cond-mat/9806029v1 
This figure "fig07.JPG" is available in "JPG" format from: http://arxiv.org/ps/cond-mat/9806029v1 
This figure "fig08.JPG" is available in "JPG" format from: http://arxiv.org/ps/cond-mat/9806029v1 
This figure "fig09.JPG" is available in "JPG" format from: http://arxiv.org/ps/cond-mat/9806029v1 
This figure "fig10.JPG" is available in "JPG" format from: http://arxiv.org/ps/cond-mat/9806029v1 
This figure "fig11.JPG" is available in "JPG" format from: http://arxiv.org/ps/cond-mat/9806029v1 
This figure "fig12.JPG" is available in "JPG" format from: http://arxiv.org/ps/cond-mat/9806029v1 
This figure "fig13.JPG" is available in "JPG" format from: http://arxiv.org/ps/cond-mat/9806029v1 
This figure "fig14.JPG" is available in "JPG" format from: http://arxiv.org/ps/cond-mat/9806029v1 
This figure "fig15.JPG" is available in "JPG" format from: http://arxiv.org/ps/cond-mat/9806029v1 
This figure "fig16.JPG" is available in "JPG" format from: http://arxiv.org/ps/cond-mat/9806029v1 
This figure "fig17.JPG" is available in "JPG" format from: http://arxiv.org/ps/cond-mat/9806029v1 
This figure "fig18.JPG" is available in "JPG" format from: http://arxiv.org/ps/cond-mat/9806029v1 